\title{
Oxford Phase 3 unicompartmental knee arthroplasty: medium-term results of a minimally invasive surgical procedure
}

\author{
Lukas A. Lisowski • Michel P. J. van den Bekerom • \\ Peter Pilot • C. Niek van Dijk · Andrzej E. Lisowski
}

Received: 7 December 2009/Accepted: 29 June 2010/Published online: 17 July 2010

(C) The Author(s) 2010. This article is published with open access at Springerlink.com

\begin{abstract}
Purpose In the last decade, a major increase in the use of and interest in unicompartmental knee arthroplasty (UKA) has developed. The Oxford Phase 3 UKA is implanted with a minimally invasive technique using newly developed instruments. The objective of this prospective study was to evaluate the outcome of UKA in patients with medial osteoarthritis of the knee in a high-volume unit.

Methods Two-hundred and forty-four UKAs were performed with a minimally invasive approach. The median age was 72 (43-91) years. The median follow-up was 4.2 years (range 1-10.4 years). Fourteen patients died, and nine were considered to be lost to follow-up, but all had a well-functioning prosthesis in situ until their last follow-up. Pain, function and health-related quality of life were evaluated pre- and postoperatively using patient- and assessor-based outcome scores, as well as radiographic evidence.

Results The mean Knee Society knee and function scores, WOMAC-scores, Oxford-score and VAS pain and satisfaction all improved. Nine knees required revision. Eleven
\end{abstract}

L. A. Lisowski - M. P. J. van den Bekerom - C. N. van Dijk Department of Orthopedic Surgery, Academic Medical Center Amsterdam, Amsterdam, The Netherlands

P. Pilot

Department of Orthopedic Surgery, Reinier de Graaf Gasthuis, Delft, The Netherlands

\section{A. E. Lisowski \\ Department of Orthopedic Surgery, Atrium Medical Centre, Kerkrade/Heerlen/Brunssum, The Netherlands}

L. A. Lisowski ( $\square)$

Het Bergske 4, 6417 GN Heerlen, The Netherlands

e-mail: lalisowski@gmail.com patients required an additional arthroscopic procedure due to persisting pain secondary to intra-articular pathology, and four patients required manipulation under anaesthesia because of limited range of motion. The 7-year cumulative survival rate of the arthroplasty was $94.4 \%$. A low incidence $(21 \%)$ of a radiolucent line beneath the tibial component was observed at 5 years of follow-up.

Conclusion This study showed a high survival rate of the Oxford Phase 3 UKA. Patient satisfaction and functional performance were also very high. Major complication rate was low; in addition, the incidence of radiolucency under the tibial component, when compared to present literature, was low. When strict indication criteria are followed, excellent, durable, and in our opinion reliable, results can be expected for this procedure.

Keywords Unicompartmental knee arthroplasty · Results $\cdot$ Survival $\cdot$ Radiolucency

\section{Introduction}

Interest in unicompartmental knee arthroplasty (UKA) for medial osteoarthritis has increased rapidly over the last two decades [42]. The main reasons for its rising popularity are the introduction of minimally invasive surgical (MIS) techniques $[31,41]$ with modified surgical equipment, the publication of the excellent medium- and long-term results of the Oxford Phase 2 arthroplasty [14, 39, 44, 45] and the well-documented improved polyethylene wear characteristics of the mobile bearing device [18, 38]. Medial osteoarthritis of the knee is considered to be a unicompartmental disease and, when left untreated, it may later progress to involve the other knee compartments [1]. This has given rise to the rationale for the treatment of only one 
compartment, either with a high tibial osteotomy (HTO) or a unicompartmental knee arthroplasty.

In the $1970 \mathrm{~s}$, the pioneers of UKA developed and introduced their first designs [11, 16, 28], but the results of these early UKAs were controversial due to high early failure rates. This changed in 1998 when Goodfellow and colleagues reported encouraging results for the unicondylar Oxford knee arthroplasty [13]. Today's opinion is that early failures are mainly attributable to a surgeon's inadequate technique and/or lack of experience with the technically demanding procedure [25, 43].

The designer group of the Oxford Phase 3 prosthesis has reported excellent results with their implant [33]. These results were reinforced by Svard and co-workers who reported favourably on a 15- and 20-year follow-up (FU) of the Oxford prosthesis [35, 44]. Although some reports show satisfactory results in shorter or long-term FU periods [21, 39, 49], others show less favourable results compared to the series of Pandit et al. and Svard et al. [10, 23, 29]. The survival and outcomes as reported in various arthroplasty registers are, however, even less satisfying [22]. This may be attributed to their lesser experience with this technique, patient selection or to the lower number of patients treated.

The purpose of the present study is to report the functional and radiological outcome of 244 consecutive Oxford Phase 3 unicompartmental knee arthroplasties performed using a MIS technique by a single orthopaedic surgeon. Our hypothesis is that results generated by an experienced high-volume surgeon treating a large number of patients will be comparable to those of the designer group, and better than those reported in the arthroplasty registers. We will report the medium-term results as previous research has shown that most complications and failures occur in the early follow-up phase, and as a result medium-term data can indicate comparable longer-term results [13]. This study describes our experiences of using the Oxford Phase 3 prosthesis implanted by a single surgeon and focuses on postoperative knee function, early complications, number and reason of revision operations, number of additional procedures, pain and radiological results.

\section{Materials and methods}

Between 1999 and 2008, 244 medial Oxford Phase 3 arthroplasties (216 patients) (Biomet Ltd., Swindon, UK) were performed in a district general hospital and form the cohort for this study. The study was uncontrolled, nonblinded and prospective. Patients' demographic details are shown in Table 1. All knees were diagnosed with medial compartment osteoarthritis. The mean preoperative tibiofemoral angle was 0.2 (SD 4.1) degrees varus. After 2001,
Table 1 Demographic baseline characteristics of 216 patients treated by means of UKA for medial compartment osteoarthritis

\begin{tabular}{|c|c|}
\hline Number of prostheses & $N=244(N=216$ patients $)$ \\
\hline Follow-up $>5$ years & $N=96$ \\
\hline Bilateral & $N=28$ \\
\hline Side & 122 right; 122 left \\
\hline Age (years), median (range) & $72(43-91)$ \\
\hline $\begin{array}{c}\text { Body mass index }\left(\mathrm{kg} / \mathrm{m}^{2}\right) \\
\text { mean } \pm \mathrm{SD}(\text { range })\end{array}$ & $28 \pm 5(18.5-52.2)$ \\
\hline $\begin{array}{l}\text { Operation time }(\min ), \\
\text { mean } \pm \mathrm{SD}(\text { range })\end{array}$ & $66 \pm 14(45-120)$ \\
\hline
\end{tabular}

due to controversial reports appearing in the literature, we considered previous HTO a contraindication for Oxford arthroplasty [45]. Prior to 2001, five patients had a HTO prior to their UKA. The strict indication criteria for unicompartmental knee arthroplasty defined by White et al. [55] and Keyes et al. [19] were followed. Osteoarthritis of the patellofemoral joint and obesity were not considered contraindications for this procedure.

Fourteen patients deceased during the study period (average 34 months postoperative), none of them as a result of the surgery. These patients were included until latest follow-up. Among these patients, one UKA was revised to a total knee arthroplasty (TKA). Nine patients did not attend the outpatient clinic for their latest FU due to general health reasons and were considered as lost to FU (LTFU). These patients or their relatives were subsequently interviewed by phone, and none of them had had a revision operation (median FU 57 months, range 14-96 months).

For this surveillance, patients' informed consent was obtained. This study was performed as routine FU, and examination was performed in accordance with generally accepted practice. Approval was obtained from our institutional review board.

\section{Surgical technique}

The cemented Oxford Phase 3 unicompartmental knee arthroplasty consists of cobalt chromium molybdenum spherical femoral and flat tibial components and a fully congruent polyethylene mobile bearing device. The MIS operation technique has been described in detail by Murray et al. [32] and was performed with newly developed instruments. These instruments not only allow better component positioning, but also create a perfect balance of the flexion and extension gap to achieve improved stability. The minimally invasive approach is the recommended standard approach for this procedure. The operation is performed under tourniquet and via a short paramedial incision running from the medial pole of the patella to the medial border of the tuberosity. This approach does not 
compromise the extensor mechanism or the suprapatellar pouch. Before cementing, pulsed lavage is used to rinse the subchondral bone. Full weight bearing was allowed postoperatively, and thromboprophylaxis was prescribed.

\section{Outcome measures}

The clinical FU consisted of physical examination, registration of pain and complications and a standard series of radiographs. Patients attended the routine FU assessments in the outpatient clinic scheduled at 6 weeks, 6 months and 1, 2, 5 and 10 years. Revision was defined as any surgical procedure that resulted in the removal or exchange of any of the arthroplasty components. Additional procedures were defined as additional operations that did not result in the removal of parts of the original arthroplasty.

Pain, function and health-related quality of life were evaluated pre- and postoperatively by patient- and assessor-based outcome scores. The WOMAC Questionnaire [3], the Knee Society Score (KSS) [17], Oxford Knee Score (OKS) [9] and postoperative visual analogue scale (VAS) for pain and satisfaction were used [54].

The accuracy of implant positioning was determined by standing anteroposterior and lateral radiographs on first outpatient review and then at routine outpatient clinic visits (including skyline views). The alignment was determined by means of the tibiofemoral angle. Osteoarthritic changes in the non-replaced compartments were graded according to the Ahlbäck classification modified by Mont et al. [30]. A fluoroscopic-centred technique was used to assess any radiolucency at the bone-cement interface above the femoral component and under the tibial component. All measurements were based on manufacturer's guidelines [32]. A radiolucent line of less than $2 \mathrm{~mm}$ width with a sclerotic line beneath the tibial component was considered to be physiological $[47,53]$.

Postoperative radiographs examining the progression of osteoarthritis in the lateral and patellofemoral (PF) compartments, the tibiofemoral angle and the presence and extent of radiolucency were investigated in 96 cases with a minimum follow-up of 5 years. Radiological examination including the fluoroscopic-centred technique was evaluated by consensus of two independent radiologists.

\section{Statistical analysis}

Data were analysed using SPSS software $\left(\operatorname{SPSS}^{\odot} 15.0\right.$, SPSS Inc., Chicago, IL, USA). The non-parametric Wilcoxon test was used for all non-parametric data. The general linear model (GLM) was used to compare the repeated measurements, a post hoc Bonferroni test was used to analyse the location of the difference, and p-values of $P<0.05$ were considered significant. Using revision of the arthroplasty for any cause as an end-point, a life-table was constructed, and rates of survival determined using the Kaplan-Meier survival analysis.

\section{Results}

The median FU was 4.2 years (range 1-10.4 years). Baseline characteristics are shown in Table 1, and outcome scores are shown in Table 2. A total of $84.3 \%$ of knees had good (70-84 points) or excellent (85-100 points) results for

Table 2 Outcome results of 216 patients treated by means of UKA for medial compartment osteoarthritis

\begin{tabular}{lcccccc}
\hline & Pre & 6 months & 12 months & 24 months & >30 months & $\begin{array}{c}\text { Latest FU total } \\
\text { (Mean FU 4.4 years) }\end{array}$ \\
\hline VAS Satisfied & & $1.1 \pm 1.6$ & $1.3 \pm 1.4$ & $1.2 \pm 1.1$ & $1.5 \pm 1.0$ & $1.5 \pm 1.1$ \\
VAS Pain & & $1.5 \pm 1.6$ & $1.4 \pm 1.4$ & $1.2 \pm 1.2$ & $1.3 \pm 1.2$ & $1.3 \pm 1.6$ \\
Oxford total & $13.0 \pm 9.8$ & $32.3 \pm 10.2^{*}$ & $36.2 \pm 9.0$ & $39.0 \pm 7.9$ & $38.3 \pm 9.1$ & $38.3 \pm 8.5$ \\
Oxford pain & $6.1 \pm 4.1$ & $13.1 \pm 4.4^{*}$ & $14.9 \pm 4.0$ & $16.6 \pm 3.6$ & $16.3 \pm 4.0$ & $16.2 \pm 3.9$ \\
Oxford function & $6.9 \pm 6.0$ & $19.2 \pm 6.2^{*}$ & $21.3 \pm 5.3$ & $22.4 \pm 4.6$ & $22.0 \pm 5.4$ & $22.1 \pm 4.9$ \\
WOMAC pain & $42.4 \pm 16.4$ & $81.6 \pm 17.4^{*}$ & $85.7 \pm 16.3$ & $88.1 \pm 16.4$ & $85.8 \pm 17.0$ & $85.8 \pm 17.0$ \\
WOMAC stiffness & $45.9 \pm 15.7$ & $70.6 \pm 19.6^{*}$ & $76.4 \pm 17.7$ & $79.0 \pm 19.6$ & $76.4 \pm 21.2$ & $76.2 \pm 19.3$ \\
WOMAC function & $44.1 \pm 15.7$ & $77.9 \pm 17.6^{*}$ & $82.0 \pm 16.8$ & $83.0 \pm 18.3$ & $81.3 \pm 18.7$ & $81.5 \pm 18.0$ \\
ROM & $121.8 \pm 10.8$ & $122.4 \pm 10.5^{*}$ & $124.7 \pm 10.3$ & $125.6 \pm 10.4$ & $128.1 \pm 9.3$ & $127.1 \pm 9.8$ \\
Tibiofemoral angle & $-0.23 \pm 4.1$ & $5.0 \pm 3.4^{*}$ & $5.0 \pm 3.2$ & $4.8 \pm 2.8$ & $4.8 \pm 3.0$ & $4.7 \pm 3.0$ \\
KSS knee & $44.2 \pm 13.7$ & $87.3 \pm 13.4^{*}$ & $90.8 \pm 9.6$ & $90.7 \pm 9.4$ & $92.3 \pm 9.3$ & $91.7 \pm 8.7$ \\
KSS function & $45.0 \pm 18.0$ & $85.5 \pm 15.9^{*}$ & $88.2 \pm 15.3$ & $85.9 \pm 17.3$ & $83.5 \pm 18.7$ & $84.7 \pm 17.8$ \\
\hline
\end{tabular}

The GLM showed that all scores increased postoperatively, $P<0.001$ for all scores. The Bonferroni post hoc test showed that the difference was only between preoperative and 6 month; afterwards the scores remained constant

* $P<0.001$ : compared preoperative with 6-month postoperative with paired t-test 
the knee score, and $74.7 \%$ had a good or excellent functional score. The general linear model revealed no significant change in scores after the 12-month FU period. The cumulative survival at 7 years was $94.4 \%$ (95\% CI 91.8-97.0) (Fig. 1).

Preoperatively, $90.1 \%$ of the patients with medial osteoarthritis of the knee had moderate (KSS 5-10 points) or severe pain (KSS 0 points). At 1-year postoperatively, 17 patients $(7.3 \%)$ had moderate or severe pain. At final FU, of the patients with a UKA in place, only $2(1 \%)$ had moderate pain.

\section{Early complications}

One fracture of the proximal tibia occurred intra-operatively. The patient was treated by continuous passive motion for 7 days followed by casting for another 4 weeks and has achieved a good outcome in regard to pain and function (125 degrees flexion, KS knee score 95 points, KS function score 100 points). In our series, no wound dehiscence, deep or superficial infection, deep venous thrombosis, pulmonary embolus or death related to the orthopaedic procedure were observed.

\section{Revisions}

Nine patients underwent a revision procedure at a mean of $33 \pm 27$ months after primary surgery. Details of the revised UKAs are summarised in Table 3.

\section{Additional procedures}

Fifteen additional procedures were performed. Four patients, with limited flexion postoperatively, required manipulation of the knee under anaesthesia (MUA) (mean 3.5 weeks after primary surgery) to improve ROM. Flexion of less than 90 degrees 3-6 weeks postoperatively was

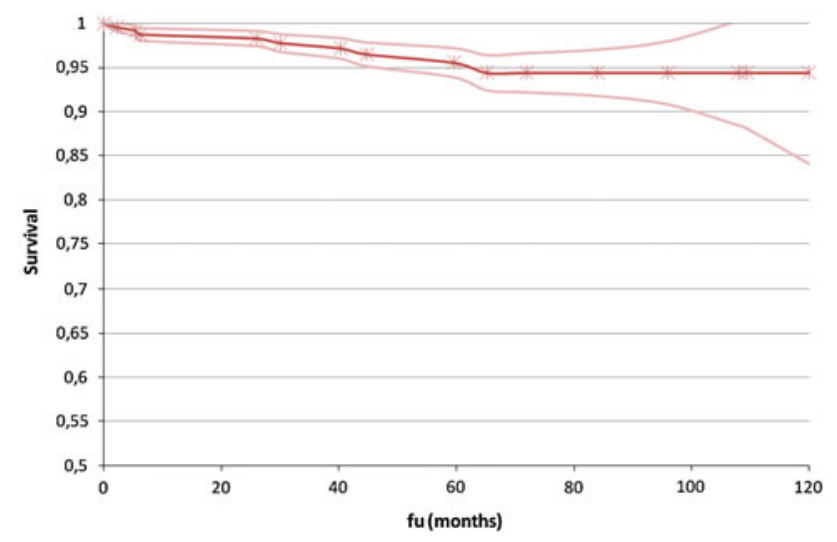

Fig. 1 Kaplan-Meier survival analysis using revision for any reason as an end-point considered an indication for MUA. At latest FU, the ROM of these patients was 123 degrees. Eleven patients underwent an arthroscopic procedure for pain related to intraarticular causes (e.g. synovitis, haemorrhage, lateral meniscal lesion, loose bodies). Three patients in this group with additional procedures were revised. The other eight arthroscopic procedures were all successful in relieving the complaints.

\section{High tibial osteotomy}

One of the five patients with a previous high tibial osteotomy was revised to a TKA. This patient still experienced pain after revision surgery to TKA. The other four patients had fair scores (range 60-69 points) and moderate function at 1-year FU (mean KS knee and function score both 61.7 points, ROM 122 degrees). These four patients eventually progressed to good and excellent scores 6.8 years after surgery (mean KSS knee: 70 points; KSS function: 83.3 points; ROM 133 degrees).

\section{Radiology}

Complete physiological radiolucency $(<2 \mathrm{~mm})$ as measured by means of the fluoroscopic-centred technique was observed in 5 tibial components. There were 15 tibial components with partial radiolucent lines. All these radiolucencies were visible at year one postsurgery and thereafter remained unchanged in extent and thickness. In two patients, pathological signs of radiolucency (i.e. a line $>2 \mathrm{~mm}$ without a thin sclerotic bordering line) beneath the tibial component were observed. These arthroplasties were still in place and functioning well at final follow-up. No radiolucency was seen between the surface of the femoral component and the bone.

Progression of medial facet PF osteoarthritis was observed in two non-symptomatic patients. The occurrence of lateral facet PF osteoarthritis was observed in four patients of whom two were symptomatic.

Five patients had a postoperative valgus angle of more than 10 degrees and were considered overcorrected. This angle was not altered at 5-year FU except in one knee, which was revised.

\section{Discussion}

The most important finding of this study was the good medium-term results of the Oxford Phase 3 UKA with a cumulative survival rate of $94.4 \%$ at 7 -year FU obtained in a high-volume district general hospital. These results are comparable with the series of Pandit et al. [34] and to the series of Robertsson et al. [43] who reported an 8-year 
Table 3 Details of the revised UKAs

\begin{tabular}{|c|c|c|c|c|c|c|}
\hline Revision & History & $\begin{array}{l}\text { Indication for } \\
\text { revision }\end{array}$ & Operative findings & $\begin{array}{l}\text { Time to } \\
\text { revision } \\
\text { (years) }\end{array}$ & Procedure & Outcome \\
\hline 1 & HTO & Pain & $\begin{array}{l}\text { PF arthritis } \\
\text { Components fixed }\end{array}$ & 3.3 & Revision to TKA & Poor $(\dagger)$ \\
\hline 2 & $\begin{array}{l}\text { ACL reinsertion } \\
10 \text { years prior }\end{array}$ & Pain & Unknown & 2.2 & $\begin{array}{l}\text { Revision to TKA } \\
\text { elsewhere }\end{array}$ & Unknown \\
\hline 3 & $\begin{array}{l}\text { Fibromyalgia } \\
\text { Polyarthritis }\end{array}$ & Pain & $\begin{array}{l}\text { PF and lateral osteoarthritis } \\
\text { Components fixed }\end{array}$ & 5.3 & $\begin{array}{l}\text { Revision to TKA } \\
\text { elsewhere }\end{array}$ & Poor \\
\hline 4 & None & $\begin{array}{l}\text { 1st Dislocation } \\
\text { 2nd dislocation }\end{array}$ & Malposition components & $\begin{array}{l}0.5 \\
0.8\end{array}$ & $\begin{array}{l}1 \text { Open bearing reposition } \\
2 \text { Revision to TKA }\end{array}$ & $\begin{array}{l}\text { Poor } \\
\text { Excellent }\end{array}$ \\
\hline 5 & None & Dislocation & $\begin{array}{l}\text { Flexion/extension gap } \\
\text { disbalance }\end{array}$ & 0.3 & $\begin{array}{l}\text { Revision to Tibial } \\
\text { Vanguard UKA }\end{array}$ & Good \\
\hline 6 & None & $\begin{array}{l}\text { Lateral compartment } \\
\text { arthritis }\end{array}$ & $\begin{array}{l}\text { Components fixed } \\
\text { MCL insufficiency } \\
\text { ACL absent }\end{array}$ & 2.5 & $\begin{array}{l}\text { Revision to Hinged TKA } \\
\text { elsewhere }\end{array}$ & Good \\
\hline 7 & Trauma & $\begin{array}{l}\text { Lateral compartment } \\
\text { arthritis }\end{array}$ & Components fixed & 6.8 & Revision to TKA & Good \\
\hline 8 & Trauma & Dislocation & Undersized bearing & 0.5 & $\begin{array}{l}\text { Open bearing reposition; } \\
\text { Thicker bearing }\end{array}$ & Good \\
\hline 9 & None & Synovitis; Cobalt allergy & $\begin{array}{l}\text { Synovitis; secondary } \\
\text { PE wear }\end{array}$ & 3.7 & $\begin{array}{l}\text { Revision to Titanium } \\
\text { AGC TKA }\end{array}$ & Good \\
\hline
\end{tabular}

survival rate of $93 \%$. The importance of high-volume units was stressed by Koskinen et al. [22] who reported high failure rates of the technically demanding Oxford arthroplasty in their Finnish Arthroplasty Register study. The results in this study compare favourably to those reported by the Finnish Arthroplasty Register which mainly reported procedures performed in low-volume clinics [22]. The average number of procedures annually in this series was 23, the same as in the series of Robertsson et al. [43].

This study reflects the experience of one single highvolume surgeon using one single implant and might not reflect the results obtained in a general community practice, with different surgeons, varying indications and numerous designs. The present study reports the outcome of patients with a medium-term FU, since functional recovery is almost complete after 1 year and does not improve significantly thereafter [34]. Almost none of the reported outcome measures improved during further FU compared to the 1-year results. Revisions for all causes occurred at a mean follow-up of 2.8 years after primary surgery. Other authors state that revisions occur mostly within 2 years after surgery [12, 20, 26, 27]. Because of these two reasons, a medium-term FU could be justified. However, as with any design one has to keep in mind that other factors (e.g. aseptic loosening, instability and progressive lateral $\mathrm{OA}$ ) that reduce the longevity of the prosthesis will influence the survival negatively with longer FU. The series by Svard et al. showed very few revisions in the second decade after the index procedure and suggests that the implant is durable in this period after implantation. So, on the other hand, the medium-term FU and a lack of control group might be considered a limitation of the study; however, complications arise usually in early follow-up [44].

In this series, two UKAs were revised due to progression of osteoarthritis of the lateral compartment. One had overcorrection at primary surgery and its alignment changed from 11 to 18 degrees valgus postoperatively. Both of these patients had a history of trauma; one sustained medial collateral ligament insufficiency and had to be revised to a hinged TKA, the other received a Vanguard TKA. In recent literature on UKAs, it has been stated that progression of arthritis in the lateral compartment is the most important reason for revision surgery [8, 10]. Kumar et al. [24] described this phenomenon as a natural progression of the disease, and Price et al. [36] reported seven revisions due to lateral OA in their series of Oxford knees. Although in our series the knee with overcorrection was revised 6.8 years after primary surgery, Weale et al. [52] showed that failures due to lateral OA often occur within 2 years, especially in overcorrected knees.

Previous studies conclude that patellofemoral osteoarthritis (PFOA) does not influence revision rate. However, they did not differentiate between medial and lateral facet degeneration [4, 24]. We agree with Weale et al. [51] that progression of medial and lateral PFOA is rare after medial 
UKA. Two of the patients with progression of medial patellofemoral facet degeneration are still doing well after 6.3 and 7.3 years FU. One patient with progression of the lateral PF facet required revision surgery. Beard et al. [2] stated that the presence of lateral PF degeneration might negatively influence the outcome of the UKA and that caution in these cases should be observed, this is contrary to the apparent lack of negative impact of worsening medial PF facet osteoarthritis. One might argue, however, that the patient with progression of lateral PF facet osteoarthritis might not have been a suitable candidate for the UKA in the first place.

The most frequent cause for revision surgery in the present series was early dislocation of the meniscal bearing $(1.2 \%)$. Lewold et al. [25] observed a dislocation of the bearing in $2.3 \%$ of their cases and three out of 688 cases $(0.4 \%)$ in the series of Pandit et al. [34] also had a bearing dislocation. Dislocation of the mobile bearing in the Oxford knee primarily occurs shortly after implantation; it can probably be attributed to a technical error during surgery. A mismatch between the tibial and femoral component, and/or size of the bearing itself, might lead to failure. In this series, two dislocations are considered to have resulted from technical errors in the surgical procedure. One of these had a malposition of the components and was revised to a TKA. The other demonstrated a flexionextension gap imbalance, and in this patient the tibial component was revised to a unilateral non-mobile tibial component. One further patient suffered a traumatic dislocation of the bearing and received a thicker insert by a conventional open procedure.

One knee was revised to a titanium AGC TKA due to a cobalt allergy (confirmed by dermatology), and secondary macroscopic polyethylene wear 3.5 years after surgery. Polyethylene wear has not been reported as a cause of failure of the Oxford knee [5, 25, 41]. No revisions were performed due to deep infection, primary polyethylene wear or loosening of the components. In contrast to the present study, the most common reason for revision in the series of Koskinen et al. [22] was aseptic loosening. As reported by others, we conclude that the indication for surgery and operative technique are predictors for failure of the arthroplasty [48].

As confirmed by others [7], arthroscopy has shown to be a successful and safe procedure post-UKA implantation for the treatment of subsequent intra-articular pathology. Psychoyios et al. [38] state that revision surgery is not always a solution, as in one of every four revisions the problem is not solved. This series suggests that patients with complaints of pain should be investigated, carefully followed up, and revision for unexplained pain must be avoided as it may not be warranted in these cases [23].
The five patients with a previous HTO required a longer time to reach their highest functional score. Vorlat et al. [50] did not show successful outcome of UKAs after HTO. Likewise, the revision rate after HTO was $28 \%$ in Rees' series [40] after a FU period of 2.9 years. One patient with a previous HTO in this series was revised from UKA to TKA. This evidence of unacceptably high failure rates emerged after the study began and was deemed reason to abandon the implantation of a UKA following HTO.

Compared to previous authors, a low incidence of radiolucency at 5-year FU was found (21\%, 20/96), for instance Pandit et al. [33] who reported radiolucent lines in $70 \%$ of their arthroplasties ( $40 \%$ complete and $60 \%$ partial). Like others, we conclude that these radiolucent lines have no clinical relevance $[6,15]$. We hypothesise that the use of thorough pulsed lavage and a dry surgical field before cementing in all the implant procedures might have played a positive role. This is further supported by the recent study of Clarius et al. [6] who also showed a lower incidence of radiolucent lines after the use of pulsed lavage in the cemented Oxford Phase 3 prosthesis after a FU of 1 year.

In patients with anteromedial osteoarthritis with persisting or progressive complaints after appropriate application of non-surgical treatment, the performance of an unicompartmental knee replacement can be recommended as long as the strict indication criteria for unicompartmental knee arthroplasty previously defined are considered $[19,55]$. Recent studies suggest that the results of unicompartmental knee arthroplasty compare well with the results of total knee arthroplasty in similar age groups [35, 46]. Long-term FU results of UKA demonstrate predictably good results comparable with those of total knee replacement $[20,33]$. With appropriate patient selection, prosthetic design and surgical technique, a surgeon can achieve good outcomes once he is well trained on its indications and technique. Although a specific number of implantations a year cannot be given, high volume for this technique is important in our opinion. With the MIS technique, patients experience a rapid recovery after the unicompartmental knee arthroplasty [37].

\section{Conclusion}

This independent prospective study showed a high survival rate of the Oxford Phase 3 UKA performed by a single surgeon. Major complication rate was low. The newly developed instruments that were introduced with the Oxford Phase 3 UKA allow the surgeon to create a good balance of the flexion and extension gap, resulting in a reduction in the risk of dislocation of the mobile bearing and overcorrection. We would not recommend the use of this procedure after HTO or in the presence of lateral facet 
PFOA. The technically demanding Oxford procedure should be performed in a high-volume unit and pulsed lavage should be used in a dry operating field with the cementing technique. Another important recommendation is that complaints of pain should be thoroughly investigated as revision for unexplained pain has been shown to produce poor results. Compared to present literature, a very low percentage of radiolucency was detected. Outcome scores after 1 year do not change significantly so a minimum FU of 1 year in this series is justified. When strict indication criteria are followed, excellent, durable, and in our opinion reliable, results can be expected for this procedure in the medium-term.

Acknowledgments The authors wish to thank Joanne BloemsaatMinekus and Matthew Easdale for their assistance. The authors did not receive any outside funding or grants in support of their research for or preparation of this work. Neither they nor a member of their immediate families received payments or other benefits or a commitment or agreement to provide such benefits from a commercial entity. No commercial entity paid or directed, or agreed to pay or direct, any benefits to any research fund, foundation, division, centre, clinical practice, or other charitable or non-profit organisation with which the authors, or a member of their immediate families, are affiliated or associated.

Open Access This article is distributed under the terms of the Creative Commons Attribution Noncommercial License which permits any noncommercial use, distribution, and reproduction in any medium, provided the original author(s) and source are credited.

\section{References}

1. Ahlback S (1968) Osteoarthrosis of the knee. A radiographic investigation. Acta Radiol Diagn (Stockh) Suppl 277:7-72

2. Beard DJ, Pandit H, Ostlere S, Jenkins C, Dodd CA, Murray DW (2007) Pre-operative clinical and radiological assessment of the patellofemoral joint in unicompartmental knee replacement and its influence on outcome. J Bone Joint Surg Br 89:1602-1607

3. Bellamy N, Buchanan WW, Goldsmith CH, Campbell J, Stitt LW (1988) Validation study of WOMAC: a health status instrument for measuring clinically important patient relevant outcomes to antirheumatic drug therapy in patients with osteoarthritis of the hip or knee. J Rheumatol 15:1833-1840

4. Callaghan JJ, Squire MW, Goetz DD, Sullivan PM, Johnston RC (2000) Cemented rotating-platform total knee replacement. A nine to twelve-year follow-up study. J Bone Joint Surg Am $82: 705-711$

5. Carr A, Keyes G, Miller R, O'Connor J, Goodfellow J (1993) Medial unicompartmental arthroplasty. A survival study of the Oxford meniscal knee. Clin Orthop Relat Res 295:205-213

6. Clarius M, Hauck C, Seeger JB, James A, Murray DW, Aldinger PR (2009) Pulsed lavage reduces the incidence of radiolucent lines under the tibial tray of Oxford unicompartmental knee arthroplasty: pulsed lavage versus syringe lavage. Int Orthop 33(6): $1585-1590$

7. Clark M, Campbell DG, Kiss G, Dobson PJ, Lewis PL (2010) Reintervention after mobile-bearing Oxford unicompartmental knee arthroplasty. Clin Orthop Relat Res 468(2):576-580
8. Collier MB, Engh CA Jr, Engh GA (2004) Shelf age of the polyethylene tibial component and outcome of unicondylar knee arthroplasty. J Bone Joint Surg Am 86:763-769

9. Dawson J, Fitzpatrick R, Murray D, Carr A (1998) Questionnaire on the perceptions of patients about total knee replacement. J Bone Joint Surg Br 80:63-69

10. Emerson RH Jr, Higgins LL (2008) Unicompartmental knee arthroplasty with the oxford prosthesis in patients with medial compartment arthritis. J Bone Joint Surg Am 90:118-122

11. Engelbrecht E (1971) Sliding prosthesis, a partial prosthesis in destructive processes of the knee joint. Chirurg 42:510-514

12. Gesell MW, Tria AJ Jr (2004) MIS unicondylar knee arthroplasty: surgical approach and early results. Clin Orthop Relat Res 428:53-60

13. Goodfellow JW, Kershaw CJ, Benson MK, O'Connor JJ (1988) The Oxford Knee for unicompartmental osteoarthritis. The first 103 cases. J Bone Joint Surg Br 70:692-701

14. Goodfellow JW, O'Connor J, Murray DW (2002) The Oxford meniscal unicompartmental knee. J Knee Surg 15:240-246

15. Gulati A, Chau R, Pandit HG, Gray H, Price AJ, Dodd CA, Murray DW (2009) The incidence of physiological radiolucency following Oxford unicompartmental knee replacement and its relationship to outcome. J Bone Joint Surg Br 91:896-902

16. Gunston FH (1971) Polycentric knee arthroplasty. Prosthetic simulation of normal knee movement. J Bone Joint Surg $\mathrm{Br}$ 53:272-277

17. Insall JN, Dorr LD, Scott RD, Scott WN (1989) Rationale of the Knee Society clinical rating system. Clin Orthop Relat Res 248:13-14

18. Kendrick BJ, Longino D, Pandit H, Svard U, Gill HS, Dodd CA, Murray DW, Price AJ (2010) Polyethylene wear in Oxford unicompartmental knee replacement: a retrieval study of 47 bearings. J Bone Joint Surg Br 92:367-373

19. Keyes GW, Carr AJ, Miller RK, Goodfellow JW (1992) The radiographic classification of medial gonarthrosis. Correlation with operation methods in 200 knees. Acta Orthop Scand 63:497-501

20. Keys GW, Ul-Abiddin Z, Toh EM (2004) Analysis of first forty Oxford medial unicompartmental knee replacement from a small district hospital in UK. Knee 11:375-377

21. Kort NP, van Raay JJ, Cheung J, Jolink C, Deutman R (2007) Analysis of Oxford medial unicompartmental knee replacement using the minimally invasive technique in patients aged 60 and above: an independent prospective series. Knee Surg Sports Traumatol Arthrosc 15:356-360

22. Koskinen E, Paavolainen P, Eskelinen A, Pulkkinen P, Remes V (2007) Unicondylar knee replacement for primary osteoarthritis: a prospective follow-up study of 1,819 patients from the Finnish Arthroplasty Register. Acta Orthop 78:128-135

23. Kuipers BM, Kollen BJ, Bots PC, Burger BJ, van Raay JJ, Tulp NJ, Verheyen CC (2010) Factors associated with reduced early survival in the Oxford phase III medial unicompartment knee replacement. Knee 17:48-52

24. Kumar A, Chambers I, Wong P (2008) Periprosthetic fracture of the proximal tibia after lateral unicompartmental knee arthroplasty. J Arthroplasty 23:615-618

25. Lewold S, Goodman S, Knutson K, Robertsson O, Lidgren L (1995) Oxford meniscal bearing knee versus the Marmor knee in unicompartmental arthroplasty for arthrosis. A Swedish multicenter survival study. J Arthroplasty 10:722-731

26. Lewold S, Robertsson O, Knutson K, Lidgren L (1998) Revision of unicompartmental knee arthroplasty: outcome in 1, 135 cases from the Swedish Knee Arthroplasty study. Acta Orthop Scand 69:469-474

27. Luscombe KL, Lim J, Jones PW, White SH (2007) Minimally invasive Oxford medial unicompartmental knee arthroplasty. A note of caution! Int Orthop 31:321-324 
28. Marmor L (1973) The modular knee. Clin Orthop Relat Res 94:242-248

29. Mercier N, Wimsey S, Saragaglia D (2009) Long-term clinical results of the Oxford medial unicompartmental knee arthroplasty. Int Orthop. doi: 10.1007/s00264-009-0869-z

30. Mont MA, Haas S, Mullick T, Hungerford DS (2002) Total knee arthroplasty for patellofemoral arthritis. J Bone Joint Surg Am 84:1977-1981

31. Murray DW, Goodfellow JW, O'Connor JJ (1998) The Oxford medial unicompartmental arthroplasty: a ten-year survival study. J Bone Joint Surg Br 80:983-989

32. Murray DW, Goodfellow JW, O'Connor JJ, Dodd CA (1999) Oxford Unicompartmental knee: manual of the surgical technique. Biomet UK Ltd, Bridgend, pp 1-40

33. Pandit H, Jenkins C, Barker K, Dodd CA, Murray DW (2006) The Oxford medial unicompartmental knee replacement using a minimally-invasive approach. J Bone Joint Surg Br 88:54-60

34. Pandit H, Jenkins C, Beard DJ et al (2009) Cementless Oxford unicompartmental knee replacement shows reduced radiolucency at one year. J Bone Joint Surg Br 91:185-189

35. Price AJ, Dodd CA, Svard UG, Murray DW (2005) Oxford medial unicompartmental knee arthroplasty in patients younger and older than 60 years of age. J Bone Joint Surg $\mathrm{Br}$ 87:1488-1492

36. Price AJ, Waite JC, Svard U (2005) Long-term clinical results of the medial Oxford unicompartmental knee arthroplasty. Clin Orthop Relat Res 435:171-180

37. Price AJ, Webb J, Topf H, Dodd CA, Goodfellow JW, Murray DW (2001) Oxford Hip and Knee Group. Rapid recovery after oxford unicompartmental arthroplasty through a short incision. J Arthroplasty 16(8):970-976

38. Psychoyios V, Crawford RW, O’Connor JJ, Murray DW (1998) Wear of congruent meniscal bearings in unicompartmental knee arthroplasty: a retrieval study of 16 specimens. J Bone Joint Surg Br 80:976-982

39. Rajasekhar C, Das S, Smith A (2004) Unicompartmental knee arthroplasty. 2- to 12-year results in a community hospital. J Bone Joint Surg Br 86:983-985

40. Rees JL, Price AJ, Lynskey TG, Svard UC, Dodd CA, Murray DW (2001) Medial unicompartmental arthroplasty after failed high tibial osteotomy. J Bone Joint Surg Br 83:1034-1036

41. Repicci JA, Eberle RW (1999) Minimally invasive surgical technique for unicondylar knee arthroplasty. J South Orthop Assoc 8:20-27
42. Ritter MA, Faris PM, Thong AE, Davis KE, Meding JB, Berend ME (2004) Intra-operative findings in varus osteoarthritis of the knee. An analysis of pre-operative alignment in potential candidates for unicompartmental arthroplasty. J Bone Joint Surg Br $86: 43-47$

43. Robertsson O, Knutson K, Lewold S, Lidgren L (2001) The routine of surgical management reduces failure after unicompartmental knee arthroplasty. J Bone Joint Surg Br 83:45-49

44. Svard U (2009) Long term results after partial knee arthroplasty with the oxford Knee. Dissertation, University of Gothenburg

45. Svard UC, Price AJ (2001) Oxford medial unicompartmental knee arthroplasty. A survival analysis of an independent series. J Bone Joint Surg Br 83:191-194

46. Tabor OB Jr, Tabor OB, Bernard M, Wan JY (2005) Unicompartmental knee arthroplasty: long-term success in middle-age and obese patients. J Surg Orthop Adv 14:59-63

47. Tibrewal SB, Grant KA, Goodfellow JW (1984) The radiolucent line beneath the tibial components of the Oxford meniscal knee. J Bone Joint Surg Br 66:523-528

48. Vardi G, Strover AE (2004) Early complications of unicompartmental knee replacement: the Droitwich experience. Knee 11:389-394

49. Verdonk R, Cottenie D, Almqvist KF, Vorlat P (2005) The Oxford unicompartmental knee prosthesis: a 2-14 year followup. Knee Surg Sports Traumatol Arthros 13:163-166

50. Vorlat P, Verdonk R, Schauvlieghe H (2000) The Oxford unicompartmental knee prosthesis: a 5-year follow-up. Knee Surg Sports Traumatol Arthrosc 8:154-158

51. Weale AE, Murray DW, Baines J, Newman JH (2000) Radiological changes five years after unicompartmental knee replacement. J Bone Joint Surg Br 82:996-1000

52. Weale AE, Murray DW, Crawford R et al (1999) Does arthritis progress in the retained compartments after 'Oxford' medial unicompartmental arthroplasty? A clinical and radiological study with a minimum ten-year follow-up. J Bone Joint Surg Br 81:783-789

53. Weale AE, Murray DW, Newman JH, Ackroyd CE (1999) The length of the patellar tendon after unicompartmental and total knee replacement. J Bone Joint Surg Br 81:790-795

54. Wewers ME, Lowe NK (1990) A critical review of visual analogue scales in the measurement of clinical phenomena. Res Nurs Health 13:227-236

55. White SH, Ludkowski PF, Goodfellow JW (1991) Anteromedial osteoarthritis of the knee. J Bone Joint Surg Br 73:582-586 